Quaderni di Geografia Cahiers de Gécongaty rataphie

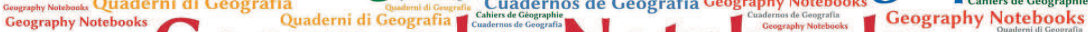
Cuadernos de Geografia Geography Notebooks $U 201000$ Cahiers de Géographi Cahiers de Géographie cahiers de Géographie Cuadernos de Geografía Cahiers de Géographie Cahiers de Géographie Cuadernos de Geografía Geography Notebooks

\author{
$4(2021)$ \\ 1 \\ Teatro di suoni. \\ Spazi acustici teatrali e territoriali
}

A cura di

Martino Mocchi, Lorena Rocca, Demis Quadri and Carlotta Sillano

EDITORIAL

Teatro di suoni per l'attaccamento ai luoghi. Uno sguardo geografico 11

Lorena Rocca

Per un teatro di suoni. Riflessioni su possibili dimensioni sonore nelle 23 creazioni site-specific di physical theatre

Demis Quadri

INTRODUCTION

Teatro di suoni. Spazi acustici teatrali e territoriali

Demis Quadri e Lorena Rocca

SPECIAL Issue

I suoni di Mantova come strumenti di interpretazione del paesaggio.

Tra turismo sostenibile ed educazione al patrimonio culturale

Valeria Pecorelli, Franca Zuccoli, Alessandra De Nicola, Enrico Squarcina

Il paesaggio sonoro campano tra contemporaneità e nuove forme

di progettualità turistica

Germana Citarella 
La narrazione spettacolarizzata del paesaggio sonoro.

Da Giuseppe Chiari a Philip K. Dick e oltre

Francesco Michi

Musica di paesaggi sonori. Enunciazione, risignificazione, comunicazione

Carlotta Sillano

Camminare per ascoltare. Partiture invisibili del territorio abitato

Elisabetta Senesi

Il paesaggio sonoro in relazione. Suono, movimento e immagini per stimolare complessità percettiva Angela Calia

Groove Fields. Understanding the Dance Floor from an Art-Based Research Perspective

Sebastian Mattbias

Il silenzio come esperienza trasformativa. L'importanza del silenzio nella meditazione e in ambito professionale

Sebastiano Caroni

Progettare il silenzio. Una lettura acustica dell'ex villaggio sanatoriale 125 Morelli a Sondalo

Martino Mocchi

Voicing One's Will. Theatre as Audio-Visual Hypotyposis of the Poetic

Michael Groneberg

Music and Clowning in Europe, 20th-21st centuries

Anna Stoll Knecht

Il paesaggio sonoro nella composizione musicale. Un percorso bibliografico

Stefano Alessandretti

\#exploreART: il labirinto di A. Pomodoro e i bambini. Un progetto di fruizione condivisa con percorsi sensoriali partecipati Alessandra De Nicola, Franca Zuccoli 


\section{OTHER EXPLORATIONS}

Il rumore lontano. Intervista a Lorena Rocca

a cura di Martino Mocchi

Re Cervo. Intervista a Antonella Astolfi

a cura di Krizia Bonaudo e Demis Quadri

Centovalli-Centoricordi. Intervista a Oliviero Giovannoni a cura di Krizia Bonaudo e Demis Quadri

Alla ricerca di un metodo: Open Space Technology 



\title{
Il paesaggio sonoro in relazione. Suono, movimento e immagini per stimolare complessità percettiva
}

\author{
Angela Calia \\ Danzatrice, Insegnante tecniche movimento e consapevolezza corporea \\ DOI: https://doi.org/10.7358/gn-2021-001-cali
}

\begin{abstract}
This article wants to explore the topic of the soundscape in the context of dance and video, especially in the site-specific creation process of dance live performances and video dance, with a brief mention to a particular type of audiovisual installations. The exploration develops starting from the soundscape that two dancing bodies produce merely moving in a dance rehearsal room, it continues with the description of a soundscape composed with sounds detected by a specific environment, and it ends with the analysis of the dance movie Car Men, by Paval Conen. The final reflections start from an example of dramaturgical use of sound in the context of film production and arrive at the description of laser drawing performance as a final example of perceptive complexity.
\end{abstract}

Keywords: soundscape; site specific; dance; video; perception.

Parole chiave: paesaggio sonoro; site specific; danza; video; percezione.

\section{INTRODUZIONE}

Questo articolo vuole esplorare il rapporto fra il suono e il movimento nel processo di realizzazione di una creazione danzata site specific, e in particolare nella creazione di un progetto di videodanza site specific. Nel farlo, si partirà da una riflessione generale sul rapporto fra suono e movimento, 
prendendo come riferimento il punto di vista di un danzatore odi una danzatrice, e partendo quindi da un rapporto estremamente esperienziale e corporeo. In particolare, il discorso si soffermerà sul rapporto fra paesaggio sonoro e creazione di una composizione coreografica, sia essa destinata alla performance live che a un progetto video.

Per paesaggio sonoro, in questo contesto, si intende fondamentalmente quell'insieme di suoni che danno vita a una sorta di colonna sonora che crea una specifica atmosfera e che riconduca a un immaginario riconoscibile nel suo complesso da uno o più suoni. Si può trattare di suoni di un ambiente naturale, del quartiere di una città di notte, di operai che lavorano in una fabbrica di tessuti. Il dato interessante non è tanto identificare esattamente il dove o il cosa, piuttosto riuscire a rilevare una connotazione dell'origine e del tipo di suono, della sua qualità e soprattutto della relazione fra i diversi suoni, che insieme creano appunto un paesaggio, come l'organico di diversi strumenti crea la musica di un'orchestra. A partire da questa prospettiva, si potrebbero distinguere i paesaggi sonori in base a una serie abbastanza ampia di caratteristiche diverse, ad esempio se i suoni sono fedelmente originali rispetto all'ambiente in cui sono stati registrati o se sono statirielaborati e ricomposti, se attengono alla sfera urbana o a quella di unbosco, ecc.

Quello che tuttavia interessa e verrà analizzato qui è il rapporto con l'elemento danza e l'elemento immagine. La riflessione infatti si svilupperà attraverso uno sguardo su diverse possibilità di approccio all'ascolto, all'analisi e alla creazione del paesaggio sonoro nella danza site specific, fino ad una breve analisi di un caso particolare di video-danza, il film Car Men di Boris Paval Conen, vincitore di diversi premie riconoscimenti tra cui il Grand Prix d'Italia, in cui la composizione del paesaggio sonoro risulta particolarmente efficace e in meravigliosa interdipendenza con le azioni danzate dei performer. Infine il testo, a partire proprio dall'esempio $\operatorname{Car} M e n$, si concluderà con una riflessione generale sulrapporto fra dimensione visiva e dimensione sonora, dapprima nel contesto filmico, poi in una interazione bidirezionale, attraverso un esempio specifico di performance di laser drawing.

\section{LA RELAZIONE FRA DANZA E PAESAGGIO SONORO NELLE CREAZIONI} SITE SPECIFIC

Qualunque danzatore e danzatrice sa quanto è intrinsecamente strutturato il rapporto di debito e credito fra musica (suono) e danza (movimen- 
to). Non c'è passo che non produca suono, neanche il più leggero, e non c'è suonoche non produca l'istinto quasi irrefrenabile di muoversi o, talvolta, di scegliere di non farlo, preferendo al movimento la stasi, alla danza agita ed esibita la presenza statica vitale e vibrante. Anche nella non azione, infatti, un corpo che danza percepisce l'intensità della vibrazione in quel movimento "mancato" e ispirato dalla relazione con la musica. Forse lo percepisce anche di più, perché nel non agire ne contiene la potenza, e il riverbero si espande in ogni cellula del corpo come le vibrazioni che lascia un sasso gettato in acqua.

Qualunque danzatore e danzatrice sa anche quanto è profondamente centrale il rapporto col silenzio e quanto incuriosisca spesso la domanda su cosa sia nata prima fra la musica e la danza. L'enigma, nell'esperienza dell'autrice, non può che essere irrisolto o irrisolvibile, perché, scavando sempre più fino all'origine di un suono o di un movimento, scopre che si originano insieme e si separano per poi spesso ritrovarsi. Anche quando un corpo che danza sceglie di andare in contrasto rispetto al suono o il suono viene prodotto in contrasto rispetto al movimento, la relazione permane, come tra due entità inseparabili per natura se pur distinte.

Creare danza in relazione o all'interno di un paesaggio sonoro può aprire vari scenari: il primo, più immediato, corrisponde al paesaggio sonoro creato dalla danza stessa, dai movimenti sul suolo, dai respiri, dagli impulsi fisici ed emotivi, dalle reazioni degli incontri dei corpi, dal peso degli stessi, dalla loro velocità e intensità di movimento.

Immaginando uno o più corpi in una sala di danza, senza alcuna musica, e in un edificio per così dire isolato, ovvero sufficientemente protetto dai suoni esterni, siano essi di contesti centrali urbani, di periferia, o anche di assoluta desolata campagna, possiamo scoprire come un'intera esplorazione danzata produca un paesaggio sonoro fervido e ricco di dettagli. Un paesaggio sonoro di corpi danzanti, un paesaggio sensoriale, dove a un orecchio attento sarà facile distinguere i suoni e associare a ognuno di essi una provenienza dei sensi: il suono del tatto dei corpi col suolo o dei corpi fra loro, dello sfregare sul pavimento o dello scivolare nel prendere spazio, del separarsi velocemente dal corpo dell'altro o del ritrovarlo stretto a sé, pelle contro pelle. Il suono del respiro e della voce, dei gemiti o della fatica, del piacere o della rabbia furiosa che può emettere un corpo che esplora se stesso nella creazione. Il suono di un bacio o di un urlo, se la narrazione danzata lo prevede o la ricerca lo scopre. Il suono della fine dalla danza, dei corpi fermi, della quiete, dell'apparente nulla.

Se basta così poco per creare un paesaggio sonoro "naturale" per 
e nelladanza, è evidente quanto possano essere vasti il potenziale di relazione fra paesaggio sonoro e movimento, e il potenziale di composizione creativa fraquesti due elementi, in cui uno può letteralmente creare l'altro, non solo come semplice ispirazione ma come parte essenziale della creazione stessa.

Questo rapporto è particolarmente significativo nei processi di creazione di danza site specific, sia che si tratti di performance dal vivo sia che si tratti di opere di video danza, ovvero immaginate, pensate e create (o talvolta riadattate partendo da un materiale già esistente) in stretta relazione con un luogo scelto. In questo caso il suono del luogo è un elemento fondamentale del processo creativo, è parte integrante dell'esplorazione e spesso costituirà la colonna sonora della performance. Si tratta di un vero e proprio dialogo fra suono e movimento in cui l'uno influenza l'altro determinando le scelte che portano alla strutturazione della composizione. Entrare a contatto con i suoni presenti in un luogo per una creazione che probabilmente nascerà, vivrà e morirà in quel luogo stesso, significa vivere quei suoni e tradurli, renderli materia di relazione con la danza, non solo da un punto di vista strettamente sonoro ma anche antropologico e culturale. Più è profondo il rapporto di indagine che la ricerca artistica opera sul luogo, più sarà rilevante l'aspetto che il suono stesso racconta di quel luogo e quindi l'uso che ne potrà fare l'artista. I quattro rintocchi di una campana possono diventare il nucleo compositivo di una partitura coreografica, il suono del ruscello che scorre può costituire il fil rouge dell'intera narrazione, le urla vivaci di bambini che irrompono imprevedibilmente per strada mentre la performance è in atto possono diventare materia di improvvisazione viva della performance stessa, inclusi istantaneamente e non ignorati, tanto quanto il suono di un clacson intermittente o di un allarme che si attiva all'esterno di un edificio.

Un ulteriore scenario interessante è quello in cui i suoni vengono registrati, estrapolati, elaborati e composti col fine di creare una partitura sonora adhoc, che magari nasce da quel luogo o che viene impiantata sul luogo ma che in qualche modo ne rileva ed enfatizza alcuni aspetti sostenendo ulteriormente la creazione danzata.

\section{CAR MEN: UN ESEMPIO DI VIDEO DANZA CON UN INTRIGANTE E COMPLESSO PAESAGGIO SONORO}

Car Men è un film ispirato alla Carmen, ben nota opera lirica di Georges Bizet, ed è il frutto della collaborazione del famoso coreografo Jíri 
Kylían, del filmmaker Boris Paval Conen e del compositore Han Otten. Il film, che ha come sfondo il paesaggio di una miniera distrutta e abbandonata della Repubblica Ceca, è interpretato da quattro danzatori, Sabine Kupferberg, Gioconda Barbuta, David Krügel e Karel Hruška, appartenenti al gruppo di danzatori senior del Dutch Dance Theater, ora non più attivo.

La narrazione ruota attorno al rapporto con la macchina Tatra 87, un'automobile molto famosa del 1937. Vediamo infatti i performer alle presecon una spesso ironica e molto poetica serie di azioni che hanno come protagonista questa auto e soprattutto vari pezzi di ferraglie che i danzatori maneggiano nel tentativo di comporre e costruire un'auto nuova. Giocano con i cerchi delle ruote come bambini spensierati, danzano con i metalli lanciandoli, spostandoli, come partner dell'azione, li usano in un gioco di seduzione e attrazione, con il sottofondo della passione della Carmen lirica.

Quello che caratterizza questo lavoro è il meraviglioso uso dei suoni metallici, combinati con la voce del canto e con suoni espressivi di risate spesso tirate all'eccesso nella loro composizione. Han Otten dunque mescola i suoni dell'oggetto principale della narrazione con quelli del luogo, con i suoni e canti dell'opera e con quelli dei protagonisti e della loro umanità grottesca e ironica: realizza una partitura sonora che esalta e si sposa con i movimenti danzati dei performer, creando un'atmosfera di contrasto fra il suono metallico e quello umano, ma anche, talvolta, rendendo il suonoumano più metallico dello stesso ferro, almeno nei suoi momenti di ripetizione quasi meccanica. Uno fra questi è la risata di una delle due coppie "alla guida dell'automobile": i due fanno solo finta di guidare e, non solo in qualche modo enfatizzano i gesti tipici di un guidatore, ma usano in maniera efficace l'espressività dei loro volti per risultare clowneschi e accattivanti allo stesso tempo. Con lo stesso principio di rielaborazione quei suoni diventano anche la colonna sonora diun'atmosfera quasi da cartone animato o da film comico muto, dove ai protagonisti accade di tutto ma non accade mai niente, poiché sono sempre intatti, allegri, quasi indistruttibili, come le leghe metalliche.

Anche in questo caso la visione comune del regista, del coreografo e del compositore sembra fare emergere una domanda sul come sia iniziata la loro creazione, e dunque se è stata la danza ad aver dato un imprinting narrativo-compositivo al suono o il suono ad aver creato le basi per il ritmo e le idee delle creazioni danzate.

Quello che è evidente è che Han Otten riesce nel compito di creare 
un paesaggio sonoro che, ascoltato a occhi chiusi, ha una chiara identità e vive di vita propria, pur essendo parte perfettamente integrata e integrante di una composizione complessa e interdisciplinare: la parte per il tutto. Prendendo in considerazione infatti i singoli linguaggi di realizzazione del film Car Men, e dunque la danza, il teatro gestuale, la musica, il video in tutte le sue ulteriori sfaccettature (la fotografia, la luce ecc.), e scegliendo poi di vivere l'opera artistica solo attraverso la dimensione sonora, come se fosse una composizione a sé stante, ci rendiamo conto che essa racchiude anche gli elementi principali degli altri linguaggi: i suoni metallici descrivono e permettono di immaginare uno scenario composto appunto da oggetti e strutture metalliche, il ritmo dei suoni permette di comprendere lo svolgimento delle azioni danzate, le risate aiutano a percepire l'atmosfera ironica e maliziosa, il canto riconduce all'opera Carmen e permette subito di riconoscere una presenza di donna e personaggio "importante" scenicamente. In qualche modo gli elementi principali narrativi sono presenti nel micro-mondo suono, tanto quanto negli altri micro-mondi artistico-espressivi, e nella loro interazione organica creano "il mondo Car Men" nel suo complesso.

\section{Il SUONO NELLA SUA FUNZIONE DRAMMATURGICA E COME STRU- MENTO DI CONNESSIONE EMPATICA}

In un processo di creazione di video danza site specific, quindi, il paesaggio sonoro risulta essere un elemento estremamente importante se si vuole realmente operare con il luogo facendo una ricerca profonda, concreta e che rispetta "la verità del luogo", anche laddove, in un secondo momento, si deciderà di stravolgere totalmente la sonorità dello stesso e creare una colonna sonora completamente differente, poiché anche in tal caso questo processo potrebbe essere significativo solo a partire dalla conoscenza del luogo stesso e del suo paesaggio sonoro reale. Potrebbe infatti essere una scelta di privazione, di contrasto, di totale immaginazione, ma che parte pur sempre da una esplorazione diretta con la realtà sonora.

Evidentemente il suono costituisce un elemento essenziale anche per ciò che concerne l'analisi e lo sviluppo, in fase creativa, del paesaggio emozionale, ed è infatti su questo aspetto che si gioca la principale partita della composizione creativa. Si tratta di un'ulteriore dimensione, rispetto a quella dell'immagine, che in qualche modo restituisce la perdita dovuta alla bidimensionalità e crea uno spazio in cui il corpo di chi è 
in scena, ma anche,e soprattutto, di chi vede e ascolta, può in un certo senso riappropriarsi diuna dimensione spaziale in cui riesce a ritrovare una collocazione.

In riferimento al prezioso lavoro svolto da Giuliana Bruno con il suo Atlante delle emozioni, e in generale prendendo spunto da una semplicissima tecnica cinematografica di utilizzo del sonoro in forma diegetica o extradiegetica, possiamo comprendere come l'effetto emotivo su chi guarda il film cambi totalmente poiché cambia il rapporto empatico che si crea fra la narrazione e lo spettatore grazie all'uso specifico del suono. Per "sonoro in forma diegetica" si intendono suoni o musica presenti e ben identificabili all'interno dell'inquadratura o della specifica scena, in modo tale che chi guarda rilevi la provenienza del suono. In sostanza dimensione visiva e dimensione uditiva della scena coincidono e accadono nello stesso momento.Per "sonoro in forma extradiegetica" si intendono suoni o musica che non provengono dall'interno dalla scena ma da qualcos'altro, come un'azione o un luogo o una dimensione emotiva che lo spettatore non vede in quel momento o di cui non è ancora consapevole. Spesso viene usata in funzione drammatica per spostare l'attenzione altrove, per anticipare eventi importanti o per raccontare qualcosa che il/la protagonista della scena ancora ignora e non vede ma che sarà determinante ai fini del racconto.

Spiegando questo punto con un esempio: assistiamo alla proiezione di un film di video danza osservando una danzatrice che si muove leggera e sorridente su un prato, mentre il paesaggio sonoro scelto dal regista è composto da suoni metallici, aggressivi, rapidi. Diventa immediatamente chiaro che in questa scena abbiamo due linee narrative come punti di vista: una data dalla dimensione visiva e l'altra da quella sonora. L'accostamento genera una sorta di contrasto o semplicemente evidenzia una non corrispondenza, e i motivi di questa scelta possono essere diversi e attengono al senso della storia: probabilmente si scoprirà che sta per accadere qualcosa alla protagonista dell'inquadratura o magari le immagini attengono alla dimensione del ricordo e i suoni alla dimensione del presente, per cui chi ricorda quelle immagini, ipotizzando un possibile scenario, è la madre della ragazza che in quel momento sta aspettando un treno, ne sente i suoni ma con la mente pensa a sua figlia.

Partendo dal presupposto che si crei una connessione empatica fra chi guarda e la scena, con quale delle due dimensioni sentiremo maggiore connessione? Che effetto provoca il contrasto fra immagine e suono? Può essere che la connessione dello spettatore o della spettatrice sia mag- 
giore in proporzione all'effetto drammatico che quel contrasto evidenzia? Cosa rivela il paesaggio sonoro rispetto all'immagine che appare così diversa da quei suoni? Si tratta in sostanza di poter usare il suono nella sua autonomia, in quanto produttore di una sua atmosfera identitaria, ma anche di metterlo in relazione in questo caso all'immagine di una donna che danza, e determinando il senso della narrazione che risulta così più ambiguo, più complesso e più intriso di possibili diversi significati.

Questa tecnica, come già evidenziato, è spesso utilizzata per creare in particolare un conflitto, una tensione, una forte connotazione emotiva. Quello che è interessante mettere in rilievo in questo contesto è che in sostanza si tratta di utilizzare il linguaggio sonoro in relazione con gli altri linguaggi, determinando la complessità vitale (e necessaria) di una narrazione che funzioni, restituendo appunto quella corporeità e complessità che a volte alla sola immagine manca o può mancare, soprattutto nel contesto delle creazioni di video danza.

E dunque, si tratta di evidenziare e valorizzare il ruolo del paesaggio sonoro in quanto tale, come un elemento autonomo ma di cui si è spesso poco consapevoli, poiché tende a viaggiare dentro di noi, ad accompagnarci nella nostra quotidianità quasi come una colonna sonora di cui non ci accorgiamo. E noto, infatti, il dato secondo cui la vista sia lo strumento corporeo di percezione maggiormente utilizzato ma a maggior ragione, nella realizzazione di un'opera artistica, la dimensione uditiva assume un valore più rilevante e diventa spesso oggetto di analisi approfondita, ricercata ed elaborata proprio in funzione di un uso drammaturgico più efficace e puntuale, spesso di grandissimo impatto ai fini dell'intreccio e sviluppo narrativo.

In questo senso, risulta più evidente comprendere come tale autonomia del paesaggio sonoro all'interno di una composizione audiovisiva site specific e, in particolare, di video danza si allinea con una maggiore potenzialità espressiva e vitale del prodotto stesso: se nelle creazioni di danza site specific dal vivo l'elemento sorpresa e/o improvvisazione può irrompere nella scena e determinare un cambiamento istantaneo ma integrato, anche nelle produzioni video vale la pena lasciare uno spazio di simile apertura all'imprevisto sonoro (e non solo sonoro) e rimanere in ascolto col luogo, anche se questo accade all'interno di una precisa sceneggiatura e partitura: tale elemento, opportunamente incluso e compreso sia nell'istante dalla regia che successivamente dal montaggio, potrà potenzialmente fornire una maggiore vitalità all'opera, un momento di sorpresa narrativa, di stupore, che all'interno di un sistema così precisa- 
mente ideato e scritto può aprire un varco nuovo e lasciare più spazio alla percezione e all'immaginazione.

Si tratta dunque di un equilibrio delicato fra scrittura e improvvisazione doveè l'elemento "dal vivo" a portare nuove possibilità esplorative nella struttura precedentemente ideata, e questo rapporto spesso risulta più avvincente e artisticamente vitale del seguire esclusivamente una delle due direzioni.

Per concludere, vale la pena citare brevemente anche un lavoro artistico audiovisivo di particolare rilevanza: il laser drawing di Alberto Novello, artista multimediale, scienziato e compositore. Si tratta di composizioni audiovisive con un rapporto bidirezionale fra suono e immagini che si sviluppano attraverso apparecchiature laser e sintetizzatori. Il processo è basato su un'interazione audiovisiva intrecciata, con effetti molto interessanti sulla percezione. Le performance di questo artista, infatti, consistono nella proiezione di disegni laser di varie forme in costante movimento con contemporanee modulazioni sonore: i suoni creano le immagini e le immagini creano i suoni, in una danza di interazione e reciprocità. Un esperimento alquanto intrigante per una videomaker, perché la supremazia dell'immagine per un attimo si ritrae e lascia maggiore spazioal suono, confermando peraltro l'interconnessione dei linguaggi: il risultato è l'affascinante manifestazione di un mondo artistico estremamente interdipendente, non gerarchico nei linguaggi e in dialogo costante, sempre foriero di stimoli interessanti e rappresentativo di una complessità percettiva artistica molto simile alla complessità percettiva della vita.

\section{RIFERIMENTI BIBLIOGRAFICI}

Bruno, G. 2015. Atlante delle emozioni. Monza: Johan \& Levi editore.

Car Men. 2016. Directed by Jiří Kylián and Boris Pavel Conen. ArtAus Musik.

\section{SitOGRAFIA}

Cinedans. http://cinedans.nl. [30/05/2020].

Alberto Novello aka Jestern. http://www.jestern.com. [18/05/2020]. 